\title{
THE PHYSICAL CONDITION OF THE PLANETS*
}

\author{
By Sir James Jeans, O.M., F.R.S.
}

$\mathrm{A}^{\mathrm{s}}$ $S$ the power of telescopes increases the astronomical horizon for ever recedes, drawing many astronomical workers with it, but leaving a few to continue their labours in the older fields. I propose here to discuss some recent work in the oldest field of all - the planets.

The physical condition of a planet depends on many factors, the most important being its distance from the sun. For we have learned in recent years that each planet gives out just about as much light and heat as it receives from the sun, and no more. Thus the planets have but little heat of their own, and are hot only to the extent that they are warmed by the sun.

Yet distance from the sun is not everything. The earth and the moon are at the same distance from the sun, so that their average temperatures are approximately the same, but their physical conditions are widely different. The moon is a dead world in which we see mountains of volcanic rock rising from plains of lava and ash, while the earth teems with life. While the earth's temperature remains fairly steady through the alternations of day and night, that of the moon rushes from one extreme to the other. According to Pettit (1940) the moon's temperature can fall from $101^{\circ} \mathrm{C}$. to $-117^{\circ} \mathrm{C}$. during an eclipse. The change of temperature is far more violent than anything we experience on earth because the moon cannot, like the earth, draw on heat stored in its soil or atmosphere. It has a surface which is probably composed of lava and ash, which are almost perfect non-conductors of heat, and it has no appreciable atmosphere-an inevitable consequence of the smallness of its mass. The earth is strong enough gravitationally to retain the molecules of its atmosphere, while the moon is not.

Bodies of intermediate mass may retain the heavier kinds of molecules, while the lighter escape. For each planet we can calculate which gases are debarred, by their lightness, from appearing in the planet's atmosphere, although to know which actually do appear we must have recourse to observation.

The light by which we see a planet is simply sunlight which has passed twice through the planet's atmosphere-once in going in and once in coming out. These journeys may produce absorption bands in the spectrum of the light, and from

* From a discourse delivered at the Royal Institution on March 25, these we can deduce the composition of the atmosphere, at least in part. There may also be some loss of light resulting from reflection at the planet's surface, for no substance reflects light perfectly. The proportion of light lost is different for different colours and by comparing the reflecting powers of the surface, colour by colour, with those of known terrestrial substances, it may be possible to identify the material of which the surface consists. Lyot has recently found that both the reflecting powers and the polarizing powers of the surfaces of Mercury, Mars and the moon are all identical with those of lava and pumice. Thus we can scarcely doubt that the surfaces of all these three bodies consist of the products of volcanic eruption in some form or other.

Let us now consider the various planets in turn, commencing with those nearest to the sun.

\section{MERCURY}

Nearest to the sun and so hottest of all the planets is Mercury. Just as the moon always presents the same face to the earth, so Mercury always presents the same face to the sun. Thus on one hemisphere of Mercury there is perpetual dayand a very hot day-while on the other hemisphere it is always night -and probably a very cold night. At the point directly under the sun, where it is always noon, the temperature is found to be about $650^{\circ}$ F., a temperature at which lead and tin are both liquid.

It is still debatable whether Mercury possesses any considerable atmosphere or not. It is the least massive of all the planets, with only one twenty-second of the substance of the earth, and its power of retaining an atmosphere is small. Under present conditions it would retain oxygen and all heavier gases, but in the past, when it was presumably hotter even than now, all gases except the very heaviest may well have streamed off into space. On the whole its surface markings are so permanent and clear-cut as to suggest that no atmosphere in fact exists. Yet Schiaparelli noticed fifty years ago that some of the surface features were occasionally seen blurred, or even obscured, as though by some sort of cloud, and Antoniadi has recently (1929) confirmed and extended his observations. As the planet could not retain molecules of water vapour, and so must be completely arid, it has been suggested 
that the clouds may consist of particles of dust, possibly set free by falls of rock. Even so there must be some sort of atmosphere to save the dust particles from immediately falling back on to the surface of the planet.

\section{VENUS}

After Mercury comes Venus, the slightly smaller twin sister of the earth. The small differences of size and mass do not produce any appreciable difference in the atmosphere-retaining capacities of the two planets, and Venus, like the earth, can retain all gases, including hydrogen. Thus if we approach the problem merely in terms of the present state of the planets, we might reasonably expect Venus to exhibit an atmosphere similar to that of the earth, although perhaps somewhat smaller in amount.

Actually the two atmospheres are found to be very different. Something of this difference is suggested by the general appearance of Venus, which presents only a continuous cloud-like surface to our view. Gerasimovič has made a study of the brightness of this cloudy envelope and of the way in which it changes with the phases of the planet, and has shown (1937) that it cannot be gaseous but must consist of large scattering particles-probably products of condensation, like the ice crystals which form the cirrus clouds in our own atmosphere. We have no means of exploring whatever atmosphere there may be below this stratum of clouds, but the "upper atmosphere" above it can of course be examined spectroscopically.

Hydrogen, nitrogen and the inert monatomic gases cannot in any case be detected spectroscopically, but oxygen and most compounds can when present in reasonable amounts. A study of the upper atmosphere of Venus reveals no oxygen and no water vapour, but an abundance of carbon dioxide. This does not necessarily mean that there is no oxygen or water vapour at all, but it means there is very little. If all the oxygen in the earth's atmosphere were collected and spread out in a layer at atmospheric pressure, this layer would be considerably over a mile in thickness, while the carbon dioxide would form a similar layer only a very few feet thick ; for the upper atmosphere of Venus, the corresponding thicknesses would be less than six feet of oxygen and more than two miles of carbon dioxide. In brief, carbon dioxide and oxygen change places. Further, St. John found that the total amount of water vapour in the upper atmosphere of Venus is certainly less than that above the highest clouds on earth.

Why should there be so great a difference in the atmospheres surrounding two such similar masses? And why in particular should the oxygen in the earth's atmosphere be free, while that in the atmosphere of Venus is combined with carbon?

As oxygen combines so eagerly with other substances, we might reasonably have expected to find but little free oxygen left in either atmosphere. It has often been suggested that the free oxygen in the earth's atmosphere may be a direct result of the vegetation on the earth's surface; that as this breaks up compounds of oxygen, carbon dioxide in particular, it sets oxygen free in the air. But this can scarcely be the whole story, since primitive life must have required, and found, some free oxygen when it first arrived on earth. Tamman has suggested (1924) that, while the earth was still hot and its solid crust not yet formed, a considerable amount of free oxygen would be produced by the thermal dissociation of water vapour. $\mathrm{He}$ calculated that if the whole of the water, ice and snow at present on earth were restored to the temperatures of this epoch, there would be enough water molecules dissociated to provide all the free oxygen at present residing in the earth's atmosphere. The free hydrogen would not, as at present, be retained but would stream off into space. No doubt some of the free oxygen would in due course combine with the solidifying rock of the earth's crust, but vegetation would afterwards come into being to restore the balance. So long as we can postulate both water vapour and vegetation, there will be no great difficulty in accounting for the presence of free oxygen.

If so, there may be no free oxygen on Venus either because there is not enough vegetation, or because there has not been enough water vapour.

As regards the first alternative, we may imagine life to have come to earth as the result of some unusual accident, some rare coincidence, or some special creation, if we prefer. If the corresponding event did not occur on Venus, the whole situation is explained ; there is no oxygen, because there is no vegetation. Or it may be that Venus has never cooled down sufficiently for life to appear. There is enough carbon dioxide in the earth's atmosphere to exert a powerful blanketing effect, checking the outward flow of radiation and so keeping the earth considerably warmer than it would otherwise be. The thousand-fold thicker layer on Venus must form a far more potent blanket, and estimates of the temperature of the lower atmosphere of Venus range from about $80^{\circ} \mathrm{C}$. to $130^{\circ} \mathrm{C}$. Atmospheric pressure on Venus may well be somewhat less than on earth, so that any water that there may be there is likely to exist in the form of steam. If so, Venus must be highly unsuited to life now and must have been even more so in the remote past.

The other possibility, which has been urged by Wildt (1940), is that Venus has always been deficient in water. As Venus and the earth were 
almost certainly formed out of the same store of matter-the outer layers of the sun-they would probably start with equal amounts of water vapour. But at a later stage, the smaller mass and higher temperature of Venus may have permitted molecules of water vapour to escape from the atmosphere of Venus, while they were still retained by the earth. If so, any small amount of oxygen set free by thermal dissociation might well be seized by the solidifying rocks, and vegetation could not then come, since there would be insufficient oxygen for it to breathe.

There would also be insufficient oxygen or ozone to screen the atmosphere from the ultra-violet rays of the sun. Wildt accordingly imagines photochemical action occurring on an extensive scale, resulting first in the dissociation of carbon dioxide and whatever water vapour there might be, and afterwards in the formation of formaldehyde $\left(\mathrm{CH}_{2} \mathrm{O}\right)$ and free oxygen. The free oxygen would continue to combine with the rocks, but the formaldehyde might be expected to remain in the atmosphere. Wildt has accordingly examined Venus spectroscopically for formaldehyde but found none (1940). He states that dry formaldehyde is stable at temperatures higher than about $80^{\circ} \mathrm{C}$. ; at lower temperatures it polymerizes into a white solid of high but unknown molecular weight. Trautz and Ufer found that the injection of a small amount of water vapour into perfectly dry formaldehyde gas produced masses of dense white cloud. Wildt suggests that the clouds we see on Venus are similar, and so consist of solid particles of hydrates of formaldehyde polymers. According to Auerbach, depolymerization into simple $\mathrm{CH}_{2} \mathrm{O}$ molecules does not occur until the temperature exceeds $200^{\circ} \mathrm{C}$, a fact which fully explains the failure of Wildt's spectroscopic search for formaldehyde. Wildt thinks it possible that Venus's atmosphere may be entirely depleted of water vapour and its surface covered with particles like those which form its clouds-a sort of formaldehyde snow.

In whatever way it may have originated, we can form a fairly accurate picture of the present state of Venus - a hot dry surface, devoid of vegetation and probably of all life such as we know on earth, surrounded by an atmosphere in which there floats a continuous layer of opaque clouds, which are probably similar in their physical, although not in their chemical, constitution to the cirrus clouds of our own atmosphere.

\section{MARS}

Crossing the earth's orbit, we come to planets which are colder than our own. Mars, which comes first, is not enormously colder, its average temperature being about $-40^{\circ} \mathrm{C}$. Actual tempera- tures are fairly widely spread about this average, those observed ranging from $+10^{\circ} \mathrm{C}$. (summer afternoon on Martian equator) to $-70^{\circ} \mathrm{C}$. (polar regions in winter).

The fact that the temperatures are not more widely spread suggests that Mars must be surrounded by a very substantial atmosphere, and observation confirms that this is so. W. H. Wright of Lick Observatory photographed the planet in infra-red light, which penetrates any atmosphere there may be and so photographs the solid body of the planet, and also with ultra-violet light, which has very little penetrating power and so photographs the surface not of the planet but of its atmosphere. He found that the ultra-violet image was measurably larger than the infra-red image, and thus obtained clear proof that Mars has an atmosphere, which he estimated to be from 50 to 60 miles high.

We still know very little of the constitution of this atmosphere. Spectroscopic analysis so far finds no definite evidence of either oxygen, carbon dioxide or water vapour. The rather insensitive test for water vapour merely shows that there cannot be a tenth as much water vapour per square yard as in our own atmosphere.

The two poles of Mars are surrounded by white areas, generally known as "polar ice caps", which diminish in size in warmer weather and disappear almost entirely in summer. They were so named from analogy with the ice-caps on our own planet, but their true nature is probably revealed by the photographs of W. H. Wright, already mentioned. The caps are found to show very clearly in ultraviolet light, but are almost invisible in infra-red light. The only possible inference would seem to be that the caps are merely atmospheric phenomena. They may well consist of clouds of small solid particles, like the clouds which cover the surface of Venus.

The want of oxygen and carbon dioxide in the atmosphere of Mars suggests that this planet also resembles Venus in not possessing vegetation of the kind we know on earth. There are, nevertheless, certain dark areas on the surface of the planet which vary unmistakably, both in colour and extent, as the seasons change. In the past these variations were frequently adduced as evidence of the presence of growing vegetation, but in the light of our present knowledge it seems more reasonable to assign meteorological causes to them-possibly the falling of rain on a desert of volcanic rock or ash.

Thus the general picture we form of Mars is that of a larger and colder moon, which, because of its somewhat greater size and mass, has retained something of an atmosphere, and may still have rains, cloud and fog to vary its appearance. 


\section{The Outer Planets}

Mars is the last planet on which a solid surface can be seen. Apart from Pluto, about which we know almost nothing, all the planets beyond Mars are much more massive than the earth, and as they are also much colder we should expect them to retain deep atmospheres, as detailed studies make it clear they do.

Wildt estimates that the atmosphere of Jupiter has a depth of 6,000 miles, with an average density of $0 \cdot 78$. For Saturn the figures are 16,000 miles and $0 \cdot 41$, so that the atmosphere occupies more than four-fifths of the whole volume of the planet.

The greater part of both atmospheres will be at a pressure of more than a million terrestrial atmospheres. Under such pressures, no known substance remains gaseous, so that what we have called the "atmospheres" of these planets must mainly consist of solids and liquids. Also, under such pressures, most substances become denser than water ; the principal exceptions are hydrogen, helium, methane $\left(\mathrm{CH}_{4}\right)$, ethane $\left(\mathrm{C}_{2} \mathrm{H}_{5}\right)$ and ammonia $\left(\mathrm{NH}_{3}\right)$. The low density of the "atmospheres" of Jupiter and Saturn suggests that they must consist largely of these substances. Hydrogen and helium, which it is not possible to detect by spectroscopic means, probably figure largely in both atmospheres, because they are abundant in the outer layers of the sun; both planets have ample gravitational power to retain them with ease, even at very high temperatures. The only substances which spectroscopic examination discloses are methane, which occurs in profusion, and ammonia, which is not found to occur in any great quantity. It is remarkable that these two gases account for absolutely the whole of the absorption of light observed in the atmospheres of Jupiter and Saturn. If other constituents are present, they are either in amounts too small to be observed or else, like hydrogen and helium, make no spectroscopic impression on light passing through them.

Still farther away from the sun come Uranus and Neptune, which prove to be merely colder miniatures of Jupiter and Saturn. The observed temperature of Jupiter is $-138^{\circ} \mathrm{C}$. and of Saturn - $153^{\circ} \mathrm{C}$. ; the temperature of Uranus is certainly lower than $-183^{\circ} \mathrm{C}$. and that of Neptune probably lower still, perhaps about $-210^{\circ} \mathrm{C}$.

In their general structure the two planets are clearly similar to Jupiter and Saturn; the spectra of their atmospheres are also similar, both showing a great abundance of methane, although ammonia has not yet been detected-possibly the extreme cold has frozen it out.
We can now try to form a physical picture of the system of planets as a whole. Leaving out Pluto, because we know nothing about it, and reserving Venus and the earth for later discussion, we see a sequence of planets-Mercury, Mars, Jupiter, Saturn, Uranus and Neptune-in which the physical conditions vary continuously as we pass along the sequence. We find heat giving place to cold, absolute aridity to an abundance of water or ice, while the atmospheres increase in depth and extent and hydrogen probably increases from nil to a large amount, existing in the form either of free hydrogen or of its compounds, especially methane.

The reason why the twin planets, Venus and the earth, do not altogether fit into this sequence is in part because they are more massive than their immediate neighbours Mercury and Mars, and so retain more atmosphere. After allowing for this, Venus fits into the sequence fairly well, but the earth still remains anomalous in having so much oxygen and water vapour in its atmosphere. This is probably a consequence of its clothing of vegetation; life has come to the earth and thrown it out of its place in the regular sequence.

Not long ago it used to be thought that the sun was continually cooling and the planets with it. What the earth now is, it used to be said, Mars must have been in the not very remote past and Venus will be in the not very remote future. If the earth is the only planet on which life now exists, Mars may nevertheless be the planet of spent life and Venus the planet of life yet to come. Our recently acquired knowledge of stellar structure and stellar evolution requires a substantial modification of this view. The sun obtains its energy from subatomic re-arrangement of its substance, light elements combining to form heavy ones, but so long as the supply of light elements does not give out entirely, the effect of these chemical changes on the sun's mechanical structure and on its outpouring of energy is extremely slight. Thus Mars must have had its present chilly temperature since those remote days when it was still warmed by the heat it had brought from the sun, and Venus will retain its present heat until the sun's supply of light elements becomes used up and it collapses into a cooler and smaller "white dwarf". This being so, the idea that life can progress along the planetary sequence is probably illusory. The earth is the planet of life because it is at the right distance from the sun, but there is no justification for thinking either that life of the kind we know on earth once existed on Mars or that it will in due course appear on Venus ; these planets are at the wrong distance from the sun. 